\title{
DISTRIBUTION DU ZOOPLANCTON LE LONG DE DEUX PETITS BASSINS CÔTIERS OUEST AFRICAINS (BIA ET AGNEBI ; CÔTE D'IVOIRE)
}

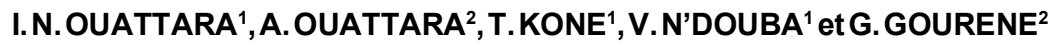 \\ ${ }^{1}$ Laboratoire d'Hydrobiologie, UFR Biosciences, Université de Cocody-Abidjan, 22 BP 582 Abidjan 22, Côte d'Ivoire. \\ E-mail : issanahoua@yahoo.fr \\ ${ }^{2}$ Laboratoire d'Environnement et de Biologie Aquatique, UFR Sciences et Gestion de l'Environnement, Université \\ d'Abobo-Adjamé, 02 BP 801 Abidjan 02, Côte d'Ivoire.
}

\begin{abstract}
RESUME
Des peuplements zooplanctoniques ont été étudiés le long de deux petits bassins côtiers ivoiriens. Ce sont la rivière Bia, sur laquelle ont été construits les barrages hydroélectriques d'Ayamé I et II, et la rivière Agnébi dépourvue de barrage sur son cours principal. Le long de la rivière Bia, d'importantes variations de la richesse spécifique et des densités zooplanctoniques moyennes ont été observées. Le zooplancton a été plus diversifié $(n=56)$ et plus abondant $\left(116675\right.$ individus $\left./ \mathrm{m}^{3}\right)$ dans le lac qu'en amont $(n=22 ; 3580$ individus $/ \mathrm{m}^{3}$ ) et en aval $\left(\mathrm{n}=16 ; 9180\right.$ individus $\left./ \mathrm{m}^{3}\right)$. En revanche, les fluctuations du nombre d'espèces et des densités moyennes du zooplancton entre les cours supérieur $\left(\mathrm{n}=11 ; 5580\right.$ individus $\left./ \mathrm{m}^{3}\right)$, moyen $\left(\mathrm{n}=22 ; 11920\right.$ individus $\left./ \mathrm{m}^{3}\right)$ et inférieur $\left(\mathrm{n}=22 ; 12280\right.$ individus $\left./ \mathrm{m}^{3}\right)$ ont été moins marquées le long de la rivière Agnébi. L'analyse du du zooplancton et des caractéristiques physiques du milieu (RDA, CANOCO) a permis de montrer que la densite du phytoplancton, la transparence et la vitesse du courant sont les facteurs les plus déterminants dans la distribution du zooplancton le long des bassins étudiés.
\end{abstract}

Mots clés : Zooplancton, distribution, rivières Bia et Agnébi, Côte d'Ivoire.

\section{ABSTRACT}

ZOOPLANKTON DISTRIBUTION ALONG TWO WEST AFRICAN SMALL RIVERS OF CÔTE D'IVOIRE (BIA AND AGNEBI)

zooplankton distribution was assessed along two Ivorian coastal basins : the river Bia on which two hydroelectric dams (Ayame I and Ayame II)have been built and the Agnebi river free of dam. There were significant differences in zooplanktonic compositions and abundance across the Bia river basin sampling sites. Zooplankton was more diversified $(n=56)$ and abundant (116675 individuals $\left./ \mathrm{m}^{3}\right)$ in Ayamé I than in upper $\left(n=22 ; 3580\right.$ individuals $\left./ m^{3}\right)$ and lower course $\left(n=16 ; 9180\right.$ individuals $\left./ m^{3}\right)$. Adversely, variations of zooplankton composition and abundance between the upstream $\left(n=11 ; 5580\right.$ individuals $\left./ \mathrm{m}^{3}\right)$, the middle stream $\left(n=22 ; 11920\right.$ individuals $\left./ m^{3}\right)$ and the lower stream $\left(n=22 ; 12280\right.$ individuals $\left./ m^{3}\right)$ of the River Agnébi were less noticeable. Combined analysis (RDA, CANOCO) of zooplankton abundance and environmental characteristics showed that phytoplankton, water transparency and water current were the environmental variables that most affect zooplankton distribution along these two coastal rivers.

Key words :Zooplankton, distribution, Bia and Agnébi rivers, Côte d'lvoire.

\section{INTRODUCTION}

L'intégrité de certaines rivières a été, ces dernières années, sérieusement menacée par la création de réservoirs hydroélectriques et agropastoraux. De nombreux travaux, ont par conséquent été entrepris pour évaluer l'impact de cette pratique sur la biodiversité des organismes aquatiques. Certaines de ces études, portant sur la composition de la communauté de poissons ont revélé entre autres, l'absence d'espèces rhéophiles dans la partie lacustre du bassin et des différences entre 
la diversité biologique des environnements lotiques et lentiques (Baijot et al., 1994 ; Gourène et al., 1999). Des différences significatives ont aussi été notées entre les caractéristiques chimiques et physiques des différents types d'écosystèmes générés par la construction du barrage ( $\mathrm{Da}$ Costa et al., 2002). Bien que les effets néfastes de la perturbation des rivières sur la biodiversité des poissons soient bien connus, les travaux concernant l'impact de ces réalisations sur les communautés zooplanctoniques demeurent rares. Par ailleurs, il est bien connu que les stratégies de conservation et de gestion durable de la biodiversité sont basées, entre autres, sur des informations complémentaires telles que la richesse d'un taxon indicateur et l'endémisme (Van Jaarsveld et al., 1998).

Depuis une vingtaine d'années, des recherches ont été conduites sur la rivière Bia, tout particulièrement sur le lac de barrage d'Ayamé I afin d'apprécier la dynamique du peuplement zooplanctonique (Yté, 1982 ; N'Douba, 1987). Toutefois, ces études ne rendent pas compte de la diversité et de la structuration de cette communauté le long de cette rivière. D'autre part, les communautés zooplanctoniques de la rivière Agnébi n'ont jusqu'à ce jour, suscité aucun intérêt, contrairement à celles de la Bia.

Ce travail a donc pour objectif d'étudier la distribution des peuplements zooplanctoniques le long des rivières Bia et Agnébi.

\section{MATERIEL ET METHODES}

\section{MILIEUD'ETUDE}

La rivière Bia, située en zone forestière, est longue de $300 \mathrm{~km}$. Elle prend sa source au Ghana et se jette dans la lagune Abi en Côte d'Ivoire (Figure 1). En 1959, l'Etat de Côte d'Ivoire a édifié sur son cours principal, le barrage hydroélectrique d'Ayamé I. Cet ouvrage a engendré trois environnements écologiquement différents : (1) l'amont (Bianouan) dont la profondeur (6,50 m maximum) dépend des phénomènes de crues et de décrues de la rivière, (2) la retenue d'eau (lac d'Ayamé I) avec une superficie de $93,2 \mathrm{~km}^{2}$ et une profondeur maximale de $30 \mathrm{~m}$, et (3) l'aval (Aboisso ; 4,3 m maximum), sous l'influence de l'ouverture ou la fermeture du barrage liée à la production d'électricité. Les sites échantillonnés se répartissent entre ces trois types de biotopes.

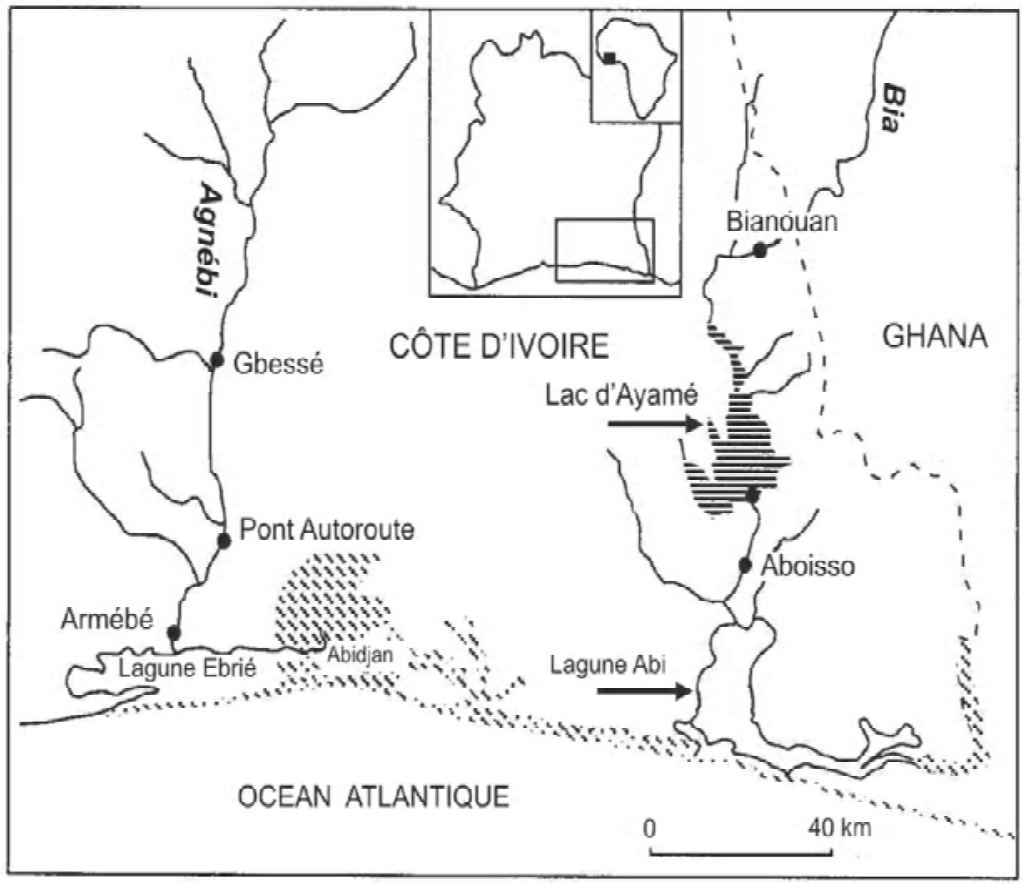

Figure 1 : Stations d'échantillonnage sur les rivières Bia et Agnébi (Côte d'Ivoire). (source : Gourène et al., 1999)

Sampling sites on Bia and Agnebi rivers (Côte d'lvoire) (source : Gourène et al., 1999). 
La rivière Agnébi, longue de 200 km et non affectée par la construction de barrage sur son cours principal, est également localisée en zone forestière. Sur cette rivière, les échantillonnages ont été effectués dans les cours supérieur (Gbessé), moyen (Pont Autoroute) et inférieur (Armébé).

Le climat des bassins des rivières Bia et Agnébi est de type équatorial humide. II comprend deux saisons sèches (juillet-août et novembre-février) et deux saisons humides (septembre-octobre et mars-juin), correspondant respectivement aux périodes de basses et hautes eaux.

\section{METHODES}

Le zooplancton a été prélevé mensuellement sur une période d'un an (de mars 1996 à février 1997). Les échantillonnages ont été effectués ( 9 h10 h) en filtrant 50 I d'eau prélevés dans les 50 premiers centimètres de la surface à l'aide d'un filet à plancton dont la partie filtrante est constituée de soie de $20 \mu \mathrm{m}$ de vide de maille. Les prélèvements $(100 \mathrm{ml})$ ont été fixés avec du formaldéhyde à $5 \%$. Les organismes zooplanctoniques ont été identifiés d'après les clés de Dussart (1980), Rey et Saint-Jean (1980) et de De Ridder (1981) et comptés sous un microscope inversé de type Leitz Diavert.

Au cours de chaque prélèvement, l'oxygène dissous, la température (oxymètre OXI 96), la conductivité (conductimètre HACH 44600), la transparence (disque de Secchi, $30 \mathrm{~cm}$ de diamètre) et la vitesse du courant ont été mesurés. Quant aux phosphates et nitrates, ils ont été dosés au moyen du spectrophotomètre DREL/2000. Des échantillons d'eau ont également été prélevés à l'aide de la bouteille hydrologique de Van Dorn en vue de dénombrer ces organismes phytoplanctoniques.

La similarité de la composition spécifique du zooplancton entre les sites échantillonnés a été appréciée à l'aide de l'indice de similarité de Sorensen (q) (Dajoz, 1985), calculé selon la formule suivante:

$q=[2 c /(a+b)] \times 100$,

où $a$ et $b$ correspondent respectivement aux nombres d'espèces observées dans les sites $A$ et $B$ et $c$, étant le nombre d'espèces communes aux deux sites.

Afin d'analyser la corrélation entre les facteurs de l'environnement et la distribution du zooplancton le long des bassins, deux matrices ont été développées. La première présente l'abondance des taxons dans les prélèvements mensuels et la seconde, les variables explicatives (variables environnementales) de toutes les stations d'échantillonnage. La relation entre les assemblages de zooplancton et les variables explicatives ont été examinées en soumettant les matrices à une analyse de redondance (RDA) à l'aide du logiciel CANOCO version 4.0 (Ter Braak et Smilauer, 1998). La RDA est une ordination de valeur propre spécialement développée pour rattacher des données écologiques multi-variées et tracer des diagrammes qui montrent à la fois la similarité basée sur les assemblages zooplanctoniques entre stations d'échantillonnage et la relation entre les densités du zooplancton, suivant l'apport des variables explicatives (Godinho et al., 1998).

Du fait de la variabilité des échantillons de zooplancton, et suivant les recommandations de Downing et al. (1987), toutes les densités prises en compte dans l'analyse en RDA ont fait l'objet d'une transformation logarithmique.

Une analyse de variance d'ordre 1 (ANOVA, Statistica 5.0), suivie d'une comparaison deux à deux des moyennes Post Hoc Comparison (LSD), a été réalisée sur la densité des groupes zooplanctoniques (copépodes, cladocères et rotifères) des sites d'échantillonnage. Les différences ont été considérées significatives au seuil de 0,05.

\section{RESULTATS}

\section{RICHESSE SPECIFIQUE DU ZOOPLANCTON}

Au total, 68 espèces de zooplancton ont été recensées, dont 30 dans la rivière Agnébi et 64 dans la rivière Bia (Tableau 1). Le long de cette dernière, le nombre de taxons a été plus élevé dans le lac de barrage d'Ayamé I (56 taxons), qu'en amont (22 taxons) et en aval (16 taxons). Contrairement à la Bia, le nombre d'espèces a varié très peu dans l'Agnébi (avec 11 espèces dans le cours supérieur et 22 espèces dans les cours moyen et inférieur).

Les indices de similarité de Sorensen, calculés en vue de comparer la composition spécifique du zooplancton des sites explorés sont présentés dans le tableau 2. Les valeurs montrent, d'une part, une similarité relativement importante entre les cours moyen et inférieur 
de l'Agnébi, entre les cours supérieur et inférieur de la Bia et entre le cours supérieur de la Bia et les cours moyen et inférieur de l'Agnébi ( $q>$ $68 \%$ ). D'autre part, de faibles similarités ont été enregistrées entre les sites lotiques des deux rivières et le lac de barrage d'Ayamé I $(q<49$ $\%)$.

\section{VARIABLES ENVIRONNEMENTALES ET DISTRIBUTIONDUZOOPLANCTON}

Le tableau 3 présente les valeurs moyennes et les écart-types des variables environnementales enregistrées dans les différentes stations des rivières Bia et Agnébi. La température de l'eau, $\mathrm{du} \mathrm{pH}$, les teneurs en phosphates $\left(\mathrm{PO}_{4}\right)$ et en nitrates $\left(\mathrm{NO}_{3}\right)$ dans les stations de la Bia avoisines celles observées dans les stations de l'Agnébi. Les eaux de ce dernier bassin sont plus pauvres en oxygène dissous et moins transparentes que celles de la Bia. On note par ailleurs, une vitesse de courant nettement plus importante en amont de la Bia que dans les autres stations.

Les données de densités moyennes du zooplancton sont présentées dans le tableau 4. Le nombre total d'organismes zooplanctoniques augmente du cours supérieur (5580 individus/ $\mathrm{m}^{3}$ ) vers le cours inférieur (12280 individus $/ \mathrm{m}^{3}$ ) dans la rivière Agnébi. Par contre, dans la rivière $\mathrm{Bia}$, ce paramètre est plus élevé dans le lac de barrage d'Ayamé I ( 116675 individus $/ \mathrm{m}^{3}$ ) et plus faible en amont du lac (3580 individus $/ \mathrm{m}^{3}$ ). Quel que soit le site d'échantillonnage, la densité des cladocères est plus faible que celle des copépodes ou des rotifères. Dans le lac de barrage d'Ayamé let dans les cours moyen et supérieur de la rivière Agnébi, les copépodes sont plus abondants que les rotifères, contrairement à l'aval, l'amont et le cours inférieur de l'Agnébi.

Les tableaux 4 et 5 montrent les résultats de la comparaison (ANOVA) des densités moyennes des copépodes, des cladocères et des rotifères entre les différents sites d'échantillonnage. Dans la rivière Bia, les échantillons du lac d'Ayamé I ont porté des diffèrences plus significatives $(p<$ $0,05)$ par comparaison à ceux des milieux lotiques de ce bassin. Aucune différence significative $(p>0,05)$ n'a été notée entre les densités moyennes des copépodes, des cladocères et des rotifères des trois sites d'échantillonnage (milieux lotiques) de la rivière Agnébi.

Les résultats de la RDA, basées sur les données de densités des organismes zooplanctoniques et les variables environnementales des différentes stations échantillonnées sur les deux rivières sont présentées sur les figures 2 et 4 . Pour ces analyses, seuls les axes 1 et 2 , ayant une forte contribution (respectivement 56,6 et $13,3 \%$ pour la Bia et 35,9 et $24,4 \%$ pour l'Agnébi), ont été pris en compte pour l'interprétation des données.

La RDA des données de la rivière Bia montre des différences entre le lac et les parties lotiques de cette rivière (Figure 2). Les échantillons du lac d'Ayamé I (groupe L), se distinguant de ceux des autres stations par de fortes valeurs de transparence, de température et de phytoplancton. Elles sont positivement corrélées avec l'axe 1. Ces échantillons sont caractérisés par de fortes densités d'espèces zooplanctoniques comprenant Ceriodaphnia affinis Lilljeborg 1900 ; C. cornuta Sars 1885 ; Filinia longiseta Ehrenberg 1834 ; Keratella cochlearis Goose 1851 ; Macrothrix goeldii Richard 1897, Monommata maculata Harring et Myers 1924, Trichocerca cylindrica Imhof 1891 et $T$. similis Wierzejski 1893. Les échantillons de l'amont et de l'aval (groupe $\mathrm{R}$ ), négativement corrélés par contre, avec l'axe 1, diffèrent de ceux du lac par de fortes valeurs de vitesse du courant et de conductivité. L'axe 2 permet de séparer les échantillons des mois secs (corrélation négative) de ceux des mois pluvieux (corrélation positive), surtout au niveau du lac d'Ayamé I.

La figure 3 montre la répartition annuelle des principales espèces dans la rivière Bia. Dans les parties fluviales de cette rivière, Ceriodaphnia cornuta et Trichocerca cylindrica sont abondantes à la fin de la grande saison des pluies. En revanche, dans la partie lacustre, Monommata maculata et Trichocerca cylindrica présentent les plus fortes densités au début de cette saison. Les densités zooplanctoniques sont nettement plus importantes dans le lac que dans les parties amont et aval.

L'analyse en RDA, basée sur les données de la rivière Agnébi (Figure 4), ne montre pas de différences nettes entre les cours supérieur, moyen et inférieur. Aucune espèce de zooplancton ne semble avoir une affinité particulière avec ces sites de prélèvement. La répartition des échantillons en fonction des saisons n'est pas marquée. Toutefois Ceriodaphnia cornuta, Trichocerca cylindrica, abondantes dans les cours moyen et inférieur, et Trichocerca similis, Ceriodaphnia cornuta dans le cours supérieur, ne sont abondantes qu'en saison des pluies (Figure 5). 
Tableau 1 : Liste des taxons zooplanctoniques récoltés dans les rivières Bia et Agnébi (Côte d'Ivoire). List of zooplankton taxa collected in the Bia and Agnébi rivers (Côte d'Ivoire).

\begin{tabular}{|c|c|c|c|c|c|c|c|}
\hline \multirow{2}{*}{ Groupes } & & \multicolumn{3}{|c|}{ Rivière Bia } & \multicolumn{3}{|c|}{ Rivière Agnébi } \\
\hline & & Aval & Lac Ayamé & Amont & Cours inférieur & cours moyen & Cours supérieur \\
\hline \multirow{6}{*}{ Copépodes } & Harpacticoïdes & & & + & + & & + \\
\hline & Mesocyclops dussarti & & + & + & + & & \\
\hline & Thermocyclops consimilis* & & + & & & & \\
\hline & Thermocyclops decipiens* & + & + & + & + & + & + \\
\hline & Thermocyclops neglectus & + & + & + & + & + & + \\
\hline & Thermodiaptomus yabensis* & & + & + & + & + & + \\
\hline \multirow{15}{*}{ Cladocères } & Acroperus elongatus & & & + & & & \\
\hline & Alona pulchella & & + & & & & \\
\hline & Alonella excisa & & & & + & & \\
\hline & Alonella nana & & + & & & & \\
\hline & Bosmina tubicen* & + & + & + & + & + & \\
\hline & Camptocercus rectirostris & + & & + & + & & \\
\hline & Ceriodaphnia affinis & & + & & & & \\
\hline & Ceriodaphnia cornuta* & + & + & + & + & + & \\
\hline & Ceriodaphnia dubia* & & + & & & & \\
\hline & Chydorus eurynotus & & + & & + & + & \\
\hline & Diaphanosoma excisum* & + & + & + & + & + & + \\
\hline & Kurtzia longirostris & + & & + & & & \\
\hline & Macrothrix goeldii* & & + & & & & + \\
\hline & Moina micrura* & & + & + & + & + & + \\
\hline & Simocephalus latirostris & & + & + & + & & \\
\hline \multirow{12}{*}{ Rotifères } & Anuraeopsis coelata & & + & & & & \\
\hline & Anuraeopsis fisa* & & + & & & & \\
\hline & Anuraeopsis navicula & & + & & & & \\
\hline & Ascomorpha ecaudis & & + & & & & \\
\hline & Asplanchna brightwelli* & & + & & & & \\
\hline & Asplanchna girodi & & + & & & & \\
\hline & Beauchampiella eudactylota & & + & & & & \\
\hline & Brachionus angularis* & & + & & & & \\
\hline & Brachionus calyciflorus* & & + & & & + & \\
\hline & Brachionus caudatus & & + & & & & \\
\hline & Brachionus falcatus* & + & + & + & & + & \\
\hline & Brachionus quadridentatus* & & + & & & & \\
\hline
\end{tabular}


Tableau 1 : (suite).

(following).

\begin{tabular}{|c|c|c|c|c|c|c|c|}
\hline & Cephalodela gibba & & + & & & & \\
\hline & Conochiloides sp. ${ }^{*}$ & & + & & & & \\
\hline & Dicranophorus epicharis* & & + & & & & \\
\hline & Epiphanes clavulata* & & + & & & & \\
\hline & Filinia longiseta* & & & + & + & + & \\
\hline & Filinia opoliensis* & & + & & + & & \\
\hline & Gastropus stylifer* & & + & & & & \\
\hline & Hexarthra intermedia* & & + & & & + & \\
\hline & Hexarthra mira & & + & & & & \\
\hline & Horaella brehmi & & & & & + & \\
\hline & Keratella cochlearis & + & + & + & & + & \\
\hline & Keratella lenzi* & + & + & + & & + & + \\
\hline & Keratella quadrata & & + & & & & \\
\hline & Keratella surrulata & & + & & & & \\
\hline & Keratella tropica* & & + & & & & \\
\hline & Lecane bulla* & + & & & & & \\
\hline & Lecane closterocerca & + & + & + & + & + & + \\
\hline Rotifères & Lecane curvicornis & & + & & & & \\
\hline & Lecane leontina & & + & + & + & + & + \\
\hline & Lecane signifera & & + & & & & \\
\hline & Monommata maculata & & & & + & & \\
\hline & Mytilina mucronata & & & + & & & \\
\hline & Philodina roscota & + & & & & & \\
\hline & Platyias leloupi* & & + & & & & \\
\hline & Platyias quadricornis & & + & & & & \\
\hline & Polyarthra vulgaris* & + & + & & + & + & \\
\hline & Rotaria sp. & & & & & + & \\
\hline & Scaridium longicaudum & & + & & + & + & \\
\hline & Synchaeta pectinata & & + & & & & \\
\hline & Testudinella patina & & + & & & & \\
\hline & Trichocerca bicrustata & & + & & & & \\
\hline & Trichocerca capucina & & + & & & & \\
\hline & Trichocerca chattoni* & + & + & + & + & + & + \\
\hline & Trichocerca cylindrica & & + & & & & \\
\hline & Trichocerca similis* & + & + & + & + & + & \\
\hline Total & 68 & 16 & 56 & 22 & 22 & 22 & 11 \\
\hline
\end{tabular}

${ }^{*}$ ) espèces signalées également par Yté (1982) dans le lac d'Ayamé I.

(*) species also recorded by Yté (1982) in Ayamé l lake. 
Tableau 2 : Indice de similarité de Sorensen (q, \%) entre les stations d'échantillonnage localisées sur les rivières Bia et Agnébi (Côte d'Ivoire).

Sorensen's similarity index (q, \%) across sampling sites located on Bia and Agnébi rivers (Côte d'Ivoire).

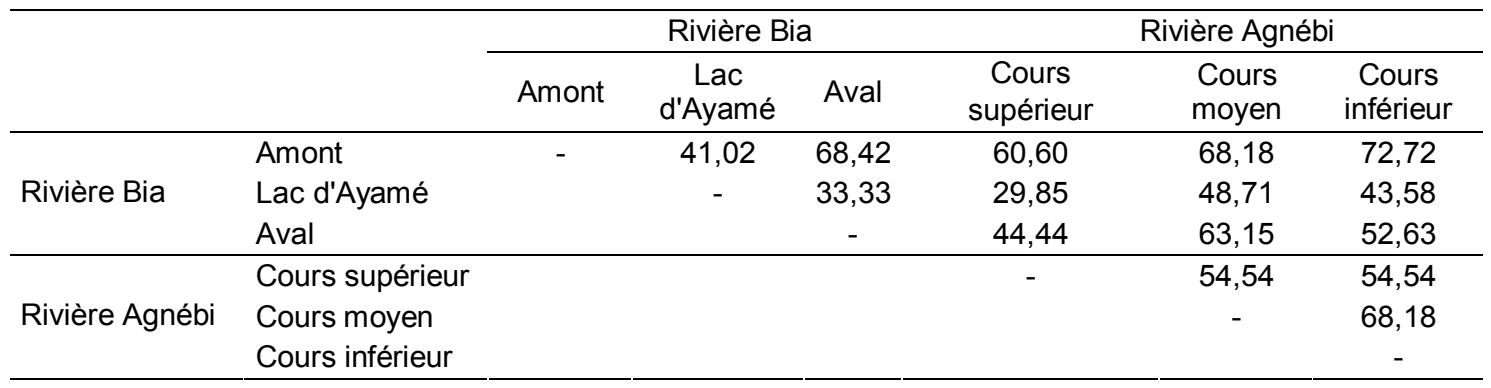

Tableau 3 : Caractéristiques physico-chimiques et densité du phytoplancton dans les sites échantillonnés sur les rivières Bia et Agnébi (Côte d'Ivoire).

Physico-chemical characteristics and phytoplankton abundance in the sampling sites located along the Bia and Agnébi rivers (Côte d'lvoire).

\begin{tabular}{|c|c|c|c|c|c|c|c|c|c|c|c|}
\hline & & & $\mathrm{T}\left({ }^{\circ} \mathrm{C}\right)$ & $\begin{array}{c}\mathrm{O} 2 \\
(\mathrm{mg} / \mathrm{l})\end{array}$ & $\begin{array}{c}\text { Cnd } \\
(\mu \mathrm{S} / \mathrm{cm})\end{array}$ & $\begin{array}{l}\text { Trans } \\
(\mathrm{cm})\end{array}$ & $\begin{array}{l}\mathrm{NO}_{3}^{-} \\
(\mathrm{mg} / \mathrm{l})\end{array}$ & $\begin{array}{l}\mathrm{PO}^{3-}{ }_{4} \\
(\mathrm{mg} / \mathrm{l})\end{array}$ & $\begin{array}{c}\text { Vit } \\
\mathrm{mm} / \mathrm{s}\end{array}$ & $\mathrm{pH}$ & $\begin{array}{c}\text { Phyt } \\
\text { cel/l.10 }\end{array}$ \\
\hline \multirow{6}{*}{ Bia } & \multirow{2}{*}{ Amont } & Moy & 25,87 & 6,23 & 115,80 & 41,25 & 0,33 & 1,31 & 408,42 & 7,50 & 0,05 \\
\hline & & ET & 0,29 & 0,38 & 4,06 & 2,46 & 0,05 & 0,20 & 8,82 & 0,25 & 0,02 \\
\hline & \multirow{2}{*}{$\begin{array}{l}\text { Lac } \\
\text { d'Ayamé }\end{array}$} & Moy & 25,98 & 6,48 & 80,38 & 109,63 & 0,31 & 1,20 & 0,00 & 7,31 & 1,62 \\
\hline & & ET & 0,34 & 0,24 & 1,95 & 8,92 & 0,05 & 0,18 & 0,00 & 0,26 & 0,50 \\
\hline & \multirow{2}{*}{ Aval } & Moy & 25,88 & 6,93 & 85,54 & 63,50 & 0,39 & 1,08 & 141,00 & 7,25 & 0,55 \\
\hline & & ET & 0,39 & 0,41 & 3,59 & 4,74 & 0,07 & 0,13 & 1,25 & 0,48 & 0,32 \\
\hline \multirow{6}{*}{ Agnébi } & \multirow{2}{*}{$\begin{array}{l}\text { Cours } \\
\text { supérieur }\end{array}$} & Moy & 24,82 & 5,70 & 142,85 & 34,75 & 0,52 & 1,19 & 131,08 & 7,39 & 0,08 \\
\hline & & ET & 0,31 & 0,13 & 6,26 & 2,74 & 0,09 & 0,10 & 3,19 & 0,41 & 0,07 \\
\hline & \multirow{2}{*}{$\begin{array}{l}\text { Cours } \\
\text { moyen }\end{array}$} & Moy & 26,27 & 4,42 & 121,37 & 45,34 & 0,41 & 1,54 & 120,83 & 7,07 & 0,13 \\
\hline & & ET & 0,43 & 0,27 & 8,05 & 3,30 & 0,06 & 0,18 & 2,84 & 0,48 & 0,06 \\
\hline & \multirow{2}{*}{$\begin{array}{l}\text { Cours } \\
\text { inférieur }\end{array}$} & Moy & 26,56 & 2,96 & 99,46 & 55,08 & 0,30 & 0,90 & 127,42 & 6,82 & 0,12 \\
\hline & & ET & 0,44 & 0,50 & 10,67 & 7,24 & 0,04 & 0,09 & 3,37 & 0,26 & 0,13 \\
\hline
\end{tabular}

Moy : moyenne ; ET : Ecart type ; $\mathrm{T}$ : température ; $\mathrm{O}_{2}$ : Oxygène dissous ; Cnd : Conductivité ; Trans : transparence ; Vit : Vitesse du courant et Phyt : phytoplancton.

Moy : mean values; ET: Standard deviation; T: temperature ; $\mathrm{O}_{2}$ : Dissolved oxygen; Cnd : Conductivity ; Trans: transparency ; Vit : water current, and Phyt : phytoplankton. 
Tableau 4 : Densité moyenne (individus $/ \mathrm{m}^{3}$ ) des groupes zooplanctoniques récoltés dans les rivières Bia et Agnébi en (Côte d'Ivoire).

Mean values of zooplankton abundance (individuals/ $\mathrm{m}^{3}$ ) in Bia and Agnébi rivers in (Côte d'Ivoire).

\begin{tabular}{lllllll}
\hline & \multicolumn{3}{c}{ Rivière Bia } & \multicolumn{3}{c}{ Rivière Agnébi } \\
\cline { 2 - 7 } & Aval & Lac Ayamé & Amont & $\begin{array}{l}\text { Cours } \\
\text { inférieur }\end{array}$ & $\begin{array}{l}\text { Cours } \\
\text { moyen }\end{array}$ & $\begin{array}{l}\text { Cours } \\
\text { supérieur }\end{array}$ \\
\hline Copépodes & $4300 \mathrm{a}$ & $70733 \mathrm{~b}$ & $1240 \mathrm{a}$ & $3820 \mathrm{a}$ & $6040 \mathrm{a}$ & $3460 \mathrm{a}$ \\
\hline Cladocères & $240 \mathrm{a}$ & $18620 \mathrm{~b}$ & $760 \mathrm{a}$ & $240 \mathrm{a}$ & $340 \mathrm{a}$ & $500 \mathrm{a}$ \\
\hline Rotifères & $4640 \mathrm{a}$ & $27322 \mathrm{~b}$ & $1580 \mathrm{a}$ & $8220 \mathrm{a}$ & $5540 \mathrm{a}$ & $1620 \mathrm{a}$ \\
\hline Total & 9180 & 116675 & 3580 & 12280 & 11920 & 5580 \\
\hline
\end{tabular}

Dans chaque ligne du tableau, les moyennes portant la même lettre, ne diffèrent pas au seuil 0.05 (ANOVA, test LSD, $\mathrm{p}>0,05)$.

In each line of the table, mean values with the same letter do not differ significantly (ANOVA, LSD test, $p>0.05$ ).

Tableau 5 : Résultats de la comparaison (ANOVA) de la densité moyenne des groupes zooplanctoniques entre les stations d'échantillonnage des rivières Bia et Agnébi (Côte d'Ivoire).

zooplankton group's abundance comparison (ANOVA) among sampling sites located on Bia and Agnébi rivers (Côte d'Ivoire).

\begin{tabular}{lccccc}
\hline & MS Effect & df Error & MS Error & F & $\mathrm{p}$ \\
\hline Copépodes & $21580.10^{4}$ & 66 & $568720.10^{4}$ & 3,794556 & $<0,05$ \\
\hline Cladocères & $841939.10^{2}$ & 66 & $126944.10^{2}$ & 6,632380 & $<0,05$ \\
\hline Rotifères & 6636073 & 66 & 495443 & 13,39420 & $<0,05$ \\
\hline
\end{tabular}

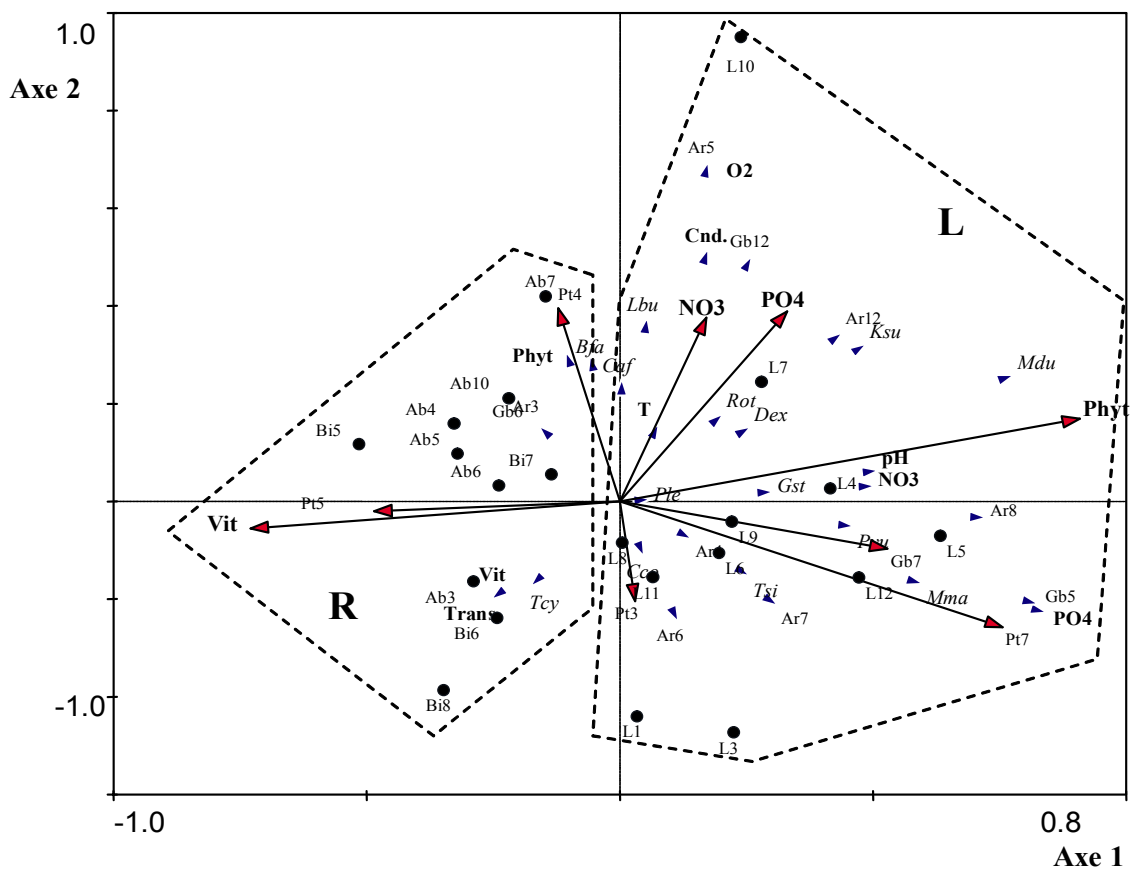

Figure 2 : Analyse de Redondance (RDA) basée sur la densité du zooplancton et les variables environnementales des sites de la rivière Bia.

Redundancy Analysis (RDA) based on zooplankton abundance and environmental characteristics of sampling sites of Bia River.

$\mathrm{L}=\operatorname{lac}(\mathrm{L} 1, \mathrm{~L} 3$ à $\mathrm{L} 12) ; \mathrm{R}=$ amont $(\mathrm{Ab3}, \mathrm{Ab7}$ à $\mathrm{Ab} 10)$ et aval (Bi5 à Bi8). $\mathrm{T}$ : température ; $\mathrm{O}_{2}$ : Oxygène dissous ; Cnd : Conductivité ; Trans : transparence ; Vit : Vitesse du courant et Phyt : phytoplancton.

$L=$ lake (L1, L3 to L12); $R=$ upstream (Ab3, Ab7 to Ab10) and lower stream (Bi5 to Bi8). T: temperature; $O_{2}$ : Dissolved oxygen; Cnd : Conductivity ; Trans: transparency ; Vit : water current and Phyt : phytoplankton. 

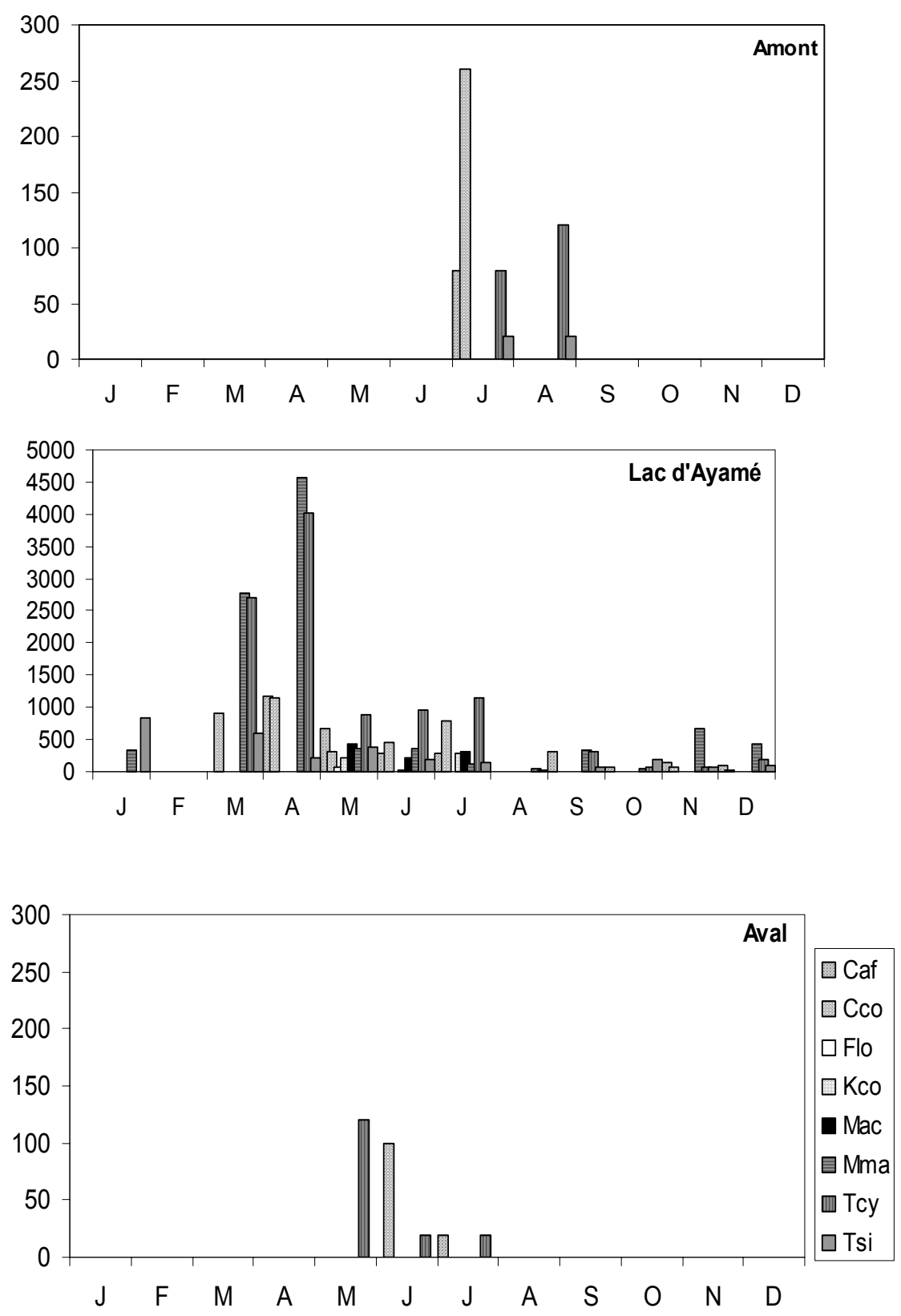

Figure 3 : Variation annuelle [janvier $(\mathrm{J})$ à décembre $(\mathrm{D})$ ] de la densité des principales espèces zooplanctoniques des sites de la rivière Bia.

Annual variation [January $(J)$ to December $(D)$ ] of abundance for major zooplankton species in sampling sites of Bia River.

Caf $=$ Ceriodaphnia affinis $; \mathrm{Cco}=$ Ceriodaphnia cornuta $;$ Flo $=$ Filinia longiseta $; \mathrm{Kco}=$ Keratella cochlearis $;$ Mac $=$ Macrothrix goeldii $;$ Mma = Monommata maculata, Tcy = Trichocerca cylindrica $;$ Tsi $=$ Trichocerca similis . 


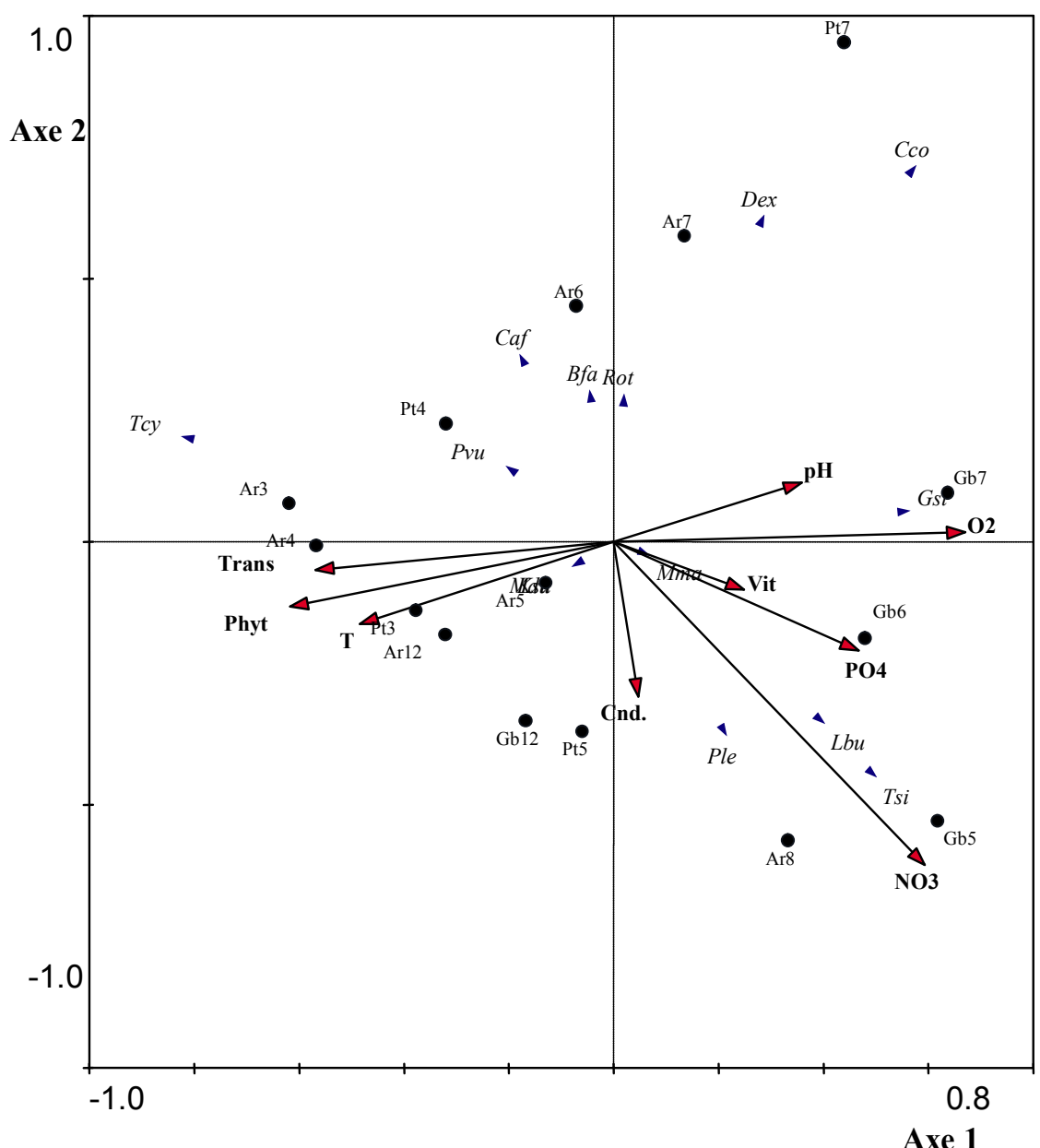

Figure 4 : Analyse de Redondance (RDA) basée sur la densité du zooplancton et les variables environnementales des sites d'échantilonnage de la rivière Agnébi.

Redundancy Analysis (RDA) based on zooplankton abundance and environmental characteristics of sampling sites on the Agnébi River.

$\mathrm{T}$ : température ; $\mathrm{O}_{2}$ : Oxygène dissous ; Cnd : Conductivité ; Trans : transparence ; Vit : Vitesse du courant et Phyt : phytoplancton. T: temperature; $\mathrm{O}_{2}$ : Dissolved oxygen; Cnd: Conductivity ; Trans: transparency ; Vit : water current and Phyt : phytoplankton.

\section{Acronymes}

Mdu : Mesocyclops dussarti ; Tde : Thermocyclops decipiens ; Tya : Thermodiaptomus yabensis ; Har : Harpacticoïdes ; Ael : Acroperus elongates ; Blo : Bosmina tubicen ; Cco : Ceriodaphnia cornuta ; Dex : Diaphanosoma excisum ; Mac : Macrothrix goeldii ; Mmi : Moina micrura ; Afi : Anuraeopsis fisa ; Bca : Brachionus calyciflorus ; Bfa : B. falcatus ; Kco : Keratella cochlearis ; Kle : K. lenzi ; Kqu : K. quadrata ; Ple : Platyias leloupi ; Gst : Gastropus stylifer; Flo : Filinia longiseta ; Fop : F. opoliensis ; Hin : Hexarthra intermedia ; Lbu : Lecane bulla ; Mma : Monommata maculata ; Slo : Scaridium longicaudum ; Pvu : Polyarthra vulgaris ; Syn : Synchaeta pectinata ; Tch : Trichocerca chattoni ; Tcy : T. cylindrica ; Tsi : T. similis ; Abr : Asplanchna brightwelli. 

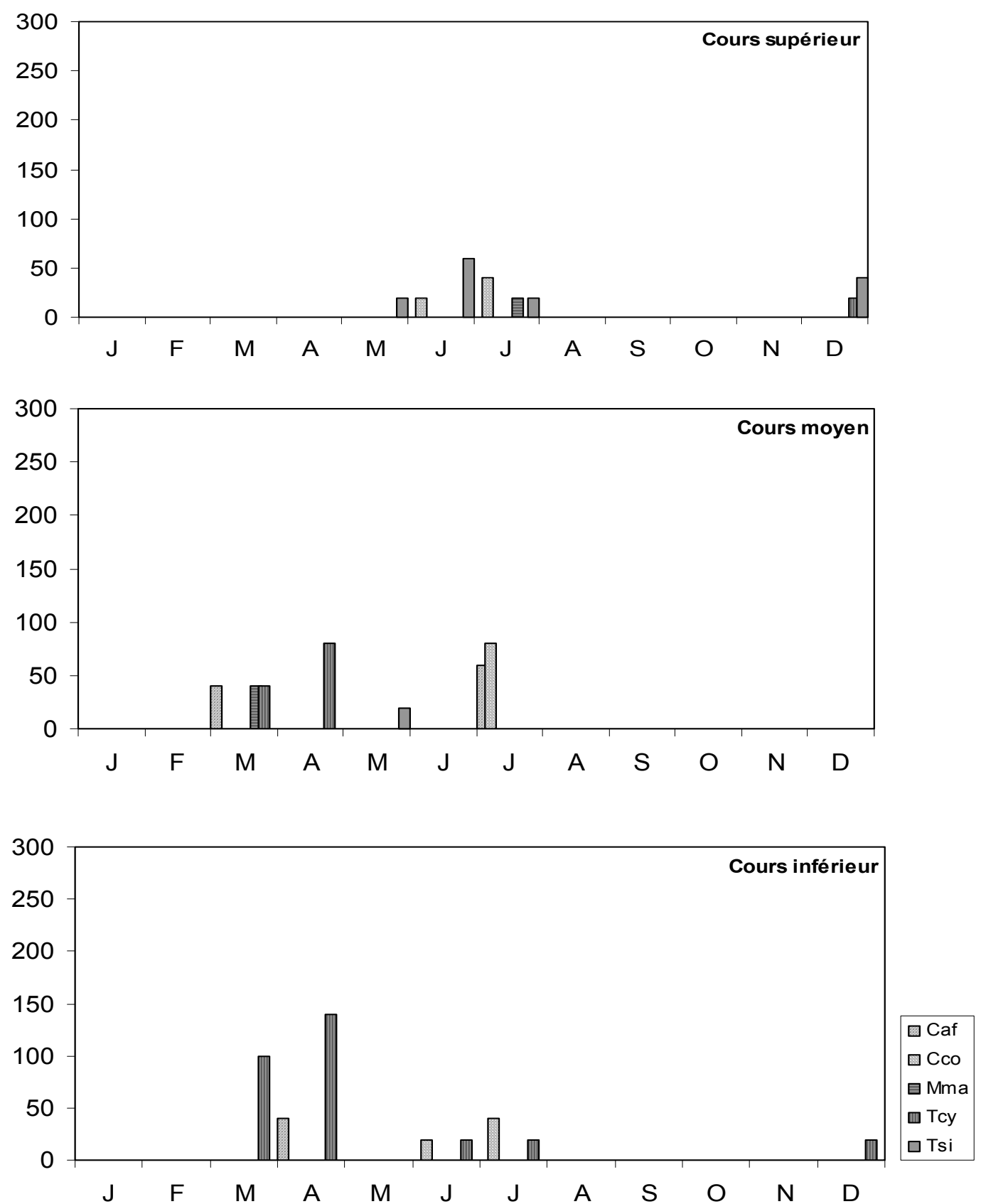

Figure 5 : Variation annuelle [janvier $(\mathrm{J})$ à décembre $(\mathrm{D})$ ] de la densité des principales espèces zooplanctoniques des sites de la rivière Agnébi.

Annual variation [January $(J)$ to December $(D)$ ] of abundance for major zooplankton species in sampling sites on the Agnebi River.

Caf $=$ Ceriodaphnia affinis $; \mathrm{Cco}=$ Ceriodaphnia cornuta $; \mathrm{Mma}=$ Monommata maculata, Tcy $=$ Trichocerca cylindrica $;$ Tsi $=$ Trichocerca similis. 


\section{DISCUSSION}

L'étude des peuplements zooplanctoniques des rivières Bia et Agnébi montre que le nombre d'espèces est plus élevé dans le lac de barrage d'Ayamé I que dans les parties fluviales des deux rivières. Ces dernières présentent, toutefois, beaucoup d'affinités dans leurs peuplements. Les fortes différences observées dans la composition des peuplements reflètent les conditions écologiquement différentes de ces deux types de milieux. Ouattara et al. (2000) ont une observation similaire au niveau des algues phytoplanctoniques de ces rivières. La richesse spécifique ( 45 taxons) notée par Yté (1982) dans le lac de barrage d'Ayamé I est plus faible que celle enregistrée dans la présente étude (56 taxons). Ces deux inventaires ont seulement en commun 29 espèces (Tableau 1). Les divergences observées pourraient, en partie, s'expliquer par la différence entre les caractéristiques des engins d'échantillonnage. En effet, Yté (1982) a utilisé un filet à plancton de $50 \mu \mathrm{m}$ de vide de maille contre $20 \mu \mathrm{m}$ dans la présente étude.

Le nombre d'espèce zooplanctonique recensé dans le lac de barrage d'Ayamé I $(n=56)$ au cours de la présente étude est supérieur à celui du lac de Buyo (surface moyenne : $920 \mathrm{~km}^{2}$; 49 espèces) (Yté et al., 1996) et à celui des petites retenues du Nord de la Côte d'Ivoire (surface moyenne : $0,03 \mathrm{~km}^{2} ; 30$ espèces) (Aka, 1998). Cependant, toutes les espèces rencontrées par ce dernier auteur ont également été échantillonnées dans le lac de barrage d'Ayamé I. La richesse spécifique dans la rivière Bia $(n=64)$ est plus élevée que dans les bassins de l'Agnébi $(n=30)$ et du Bandama (Côte d'Ivoire) (1000 km de long ; 53 espèces) (De Ridder et Pourriot, 1984). La différence entre les inventaires du zooplancton de la présente étude dans les rivières Bia et Agnébi et ceux des auteurs sus-mentionnés pourrait être liée à l'intensité des efforts d'échantillonnage. L'effort d'échantillonnage (Dumont, 1994), la taille de la retenue d'eau (Ivanova, 1987 ; Dodson et SilvaBriano, 1996 ; Aka, 1998) et les variables environnementales (Manca et Armiraglio, 2002) constituent des facteurs pouvant influencer la distribution du zooplancton.

Les données de densité et de distribution du zooplancton montrent des différences importantes entre les sites échantillonnés le long de la rivière $\mathrm{Bia}$ : le zooplancton est très abondant et plus diversifié dans le lac qu'en amont et en aval. En revanche, de telles variations ne sont pas observées dans la rivière Agnébi, non barrée.

Les variations observées dans la rivière Bia pourraient refléter la nature différente des conditions du milieu des sites échantillonnés. En effet, les résultats de la RDA montrent que les variables fortement associées à la ségrégation des assemblages sont la vitesse du courant et la conductivité (plus élevées dans les parties lotiques), la transparence, la température et la densité du phytoplancton (plus importantes dans le lac). De telles observations ont été également faite par plusieurs auteurs (Dadzie, 1980 ; Threlkeld et Choinsk, 1985 ; Betsil et Van den Avyle, 1994 ; Nogueira, 2001) qui ont montré que la construction de barrages sur un cours d'eau entraîne de profonds changements des variables chimiques, de la vélocité du courant, du substrat et des organismes aquatiques du milieu originel.

En milieu fluvial, le renouvellement permanent des eaux ne favorise pas l'abondance du zooplancton. En effet, le courant provoque une forte turbidité qui, en diminuant la pénétration de la lumière dans la colonne d'eau, réduit la croissance du phytoplancton. Ce qui limite le développement du zooplancton (Ouattara et al., 2001).

Le passage d'un système lotique à écoulement plus ou moins rapide à un système lacustre, plus calme, favorise le développement du plancton (Ouattara et al., 2001). Dans un tel système, la concentration en nutriments est plus élevée, la vitesse du courant faible, la température est plus stable et les eaux moins turbides (Richardson et Mackay, 1991). Ces observations pourraient justifier en partie la forte densité du zooplancton dans le lac d'Ayamé I. Ce développement pourrait également s'expliquer par un développement important de phytoplancton liée à la disponibilité des éléments nutritifs $\left(\mathrm{NO}_{3}\right.$ et $\left.\mathrm{PO}_{4}\right)$ et à une forte transparence des eaux, due à la sédimentation des particules lourdes en suspension. Relativement à l'évolution saisonnière des densités du zooplancton, on observe une variation plus ou moins importante dans le lac, les maximums se produisant en début de saison de pluies (avrilmai) et les minimums en saison sèche (janviermars). Ces périodes coïncident respectivement avec celles des fortes et faibles densités du phytoplancton dans le lac d Ayamé I (Ouattara 
et al., 2001). En revanche, dans les parties fluviales de la Bia et dans l'ensemble des sites de l'Agnébi, la variation saisonnière est moins marquée.

\section{CONCLUSION}

La distribution du zooplancton dans les deux bassins côtiers ivoiriens (rivières Bia et Agnébi) a été abordée pour la première fois. Les résultats montrent que le nombre d'espèces zooplanctoniques de la rivière Bia est plus élevé que celui de la rivière Agnébi. De plus, dans la Bia, les densités sont plus élevées dans le milieu lacustre, comparativement aux milieux fluviaux. En revanche, aucune variation nette n'a été notée dans la rivière Agnébi. La distribution des espèces zooplanctoniques, en relation avec les variables environnementales des différents milieux étudiés, a montré que la vitesse du courant, la transparence, la conductivité et la densité du phytoplancton sont les facteurs qui influencent le plus la répartition du zooplancton.

\section{REMERCIEMENTS}

Cette étude s'inscrit dans le cadre d'un projet VLIR sur «l'Évaluation de la diversité des poissons d'un bassin côtier ouest africain après la construction d'un barrage». Les auteurs tiennent à remercier le personnel du Projet VLIR des universités de Cocody-Abidjan et d'Abobo-Adjamé (Côte d'Ivoire) pour sa contribution au travail de terrain. Ils remercient également le Professeur Luc Brendonck (Katholieke Universiteit, Leuven) pour ses commentaires et le Professeur Dumond H. (Laboratoire d'Ecologie Animale, zoogéographie et conservation de la nature, Belgique) pour son aide à l'identification des taxons zooplanc-toniques.

\section{REFERENCES}

Aka N. M. 1998. Ecologie planctonique des petites retenues du Nord de la Côte d'Ivoire. mém. DEA, Université d'Abobo-Adjamé (Côte d'Ivoire), $61 \mathrm{p}$.

Baijot E., J. Moreau et S. Bouda. 1994. Aspects hydrobiologiques et piscicoles des retenues d'eau en zone soudano-sahélienne. CTA (ACP/CEE), Ede, 250 p.
Betsil R. K. and M. J. Van den Avyle. 1994. Spatial heterogeneity of reservoir zooplankton : a matter of timing ? Hydrobiologia 277 : $63-70$.

Da Costa K. S., K. Traoré and W. A. Yté. 2002. Potential species for fishery enhancement in Lake Faé, Côte d'Ivoire. In I. G. Cowx (Ed.). Management and Ecology of Lake and Reservoir Fisheries. Fishing News Books, University of Hull : pp $344-352$.

Dadzie S. 1980. Recent change in the fishery of a new tropical man-made lake, Lake Kamburu (Kenya). J. Fish Biol. 16 : $361-367$.

Dajoz R. 1985. Précis d'écologie. Dunod Paris, $505 \mathrm{p}$.

De Ridder M. 1981. Rotifères : Résultats scientifiques, Exploration Hydrobiologique du bassin du lac Bangweulu et du Luapula. Ann. Sc. Zool. Mus. R. Afr. Centr. 11 : 1 - 191.

De Ridder M. et R. Pourriot. 1984. Peuplement en rotifères du bassin du Bandama (Côte d'lvoire). Rev. Hydrobio. trop. 17 : 287 - 294.

Dodson S. I. et M. Silva-Briano. 1996. Crustacean zooplankton species and associations in reservoirs and ponds of Aguascalientes state, Mexico. Hydrobiologia 325 : $163-172$.

Downing J. A., M. Perusse and Y. Frenette. 1987. Effect of interreplicate variance on zooplankton sampling design and data analysis. Limnol. Oceanogr. 32 : 673 - 680.

Dumont H. J. 1994. On the diversity of the Cladocera in the tropics. Hydrobiologia $272: 27-38$.

Dussart B. H. 1980. Copépodes. In J. R. Durand et C. Lévêque (Eds.). Flore et faune aquatiques de l'Afrique sahelo-soudanienne, Tome I. ORSTOM, Paris : pp 333 - 356.

Godinho F. N., M. T. Ferreira and M. I. P. Castro. 1998. Fish assemblage composition in relation to environmental gradients in Portuguese reservoirs. Aquat. Living Resour. 11 : 325 - 334.

Gourène G., G. G. Teugels, B. Hugueny et D. F. E. Thys Van den Audenaerde. 1999. Évaluation de la diversité ichtyologique d'un bassin ouest africain après la construction d'un barrage. Cybium 23 (2) : 147 - 160.

Ivanova M. B. 1987. Relationships between zooplankton development and environmental conditions in different types of lakes in the zone of temperate climates. Inter. Rev. Ges. Hydrobiol. 72 : 669 - 684. 
Manca M. and M. Armiraglio. 2002. Zooplankton of 15 lakes in the Southern Central Alps : comparison of recent and past (pre-ca 1850 AD) communities. J. Limnol. 61 (2) : $225-231$.

N'Douba V. 1987. Contribution à l'étude des relations trophiques dans les étangs et lacs de Côte d'Ivoire : cas des peuplements de copépodes. Thèse de Doctorat $3^{\mathrm{e}}$ cycle, Université Nationale de Côte d'Ivoire, $126 \mathrm{p}$.

Nogueira M. G. 2001. Zooplankton composition, dominance and abundance as indicators of environmental compartmentalization in Jurumirim Reservoir (Paranapanema River), Sao Paulo, Brazil. Hydrobiologia $455: 1-18$.

Ouattara A., N. Podoor et G. Gourène. 2001. Etudes préliminaires de la distribution spatiotemporelle du phytoplancton dans un système fluvio-lacustre africain (bassin $\mathrm{Bia}$, Côte d'Ivoire). Hydroécol. Appl. 13 (1) : $113-132$.

Ouattara A., N. Podoor, G. G. Teugels et G. Gourène. 2000. Les micro-algues de deux cours d'eau (Bia, Agnébi) de Côte d'Ivoire. Systematic and Geography of plant, 70 (2) : $46-70$.

Rey J. et L. Saint-Jean. 1980. Brachiopodes (Cladocères). In J. R. Durand et C. Lévêque (Eds.). Flore et faune aquatiques de
l'Afrique sahelo-soudanienne, Tome I. ORSTOM, Paris : pp 307 - 332.

Richardson J. S. and R. J. Mackay. 1991. Lake outlets and the distribution of filter feeders : an assessment of hypotheses. Oikos 62 : $370-380$.

Ter Braak C. J. F. and P. Smilauer. 1998. CANOCO Reference manual and user's guide to Canoco for Windows (version 4). Center for Biometry Wageningen, $351 \mathrm{p}$.

Threlkeld S. and E. Choinsk. 1985. Plankton in a rapidly flushed impoundment spacial distribution, population dynamics and community structure. Mem. Istit. It. Idrobiol. $43: 105-118$.

Van Jaarsveld A. S., S. Freitag, S. Chown, C. Muller, S. Koch, H. Hull, C. Bellamy, M. Krüger, S. Endrödy-Younga, M. W. Mansell and C. H. Scholtz. 1998. Biodiversity assessment and conservation strategies. Science 279 : 2106 - 2108.

Yté W. A. 1982. Contribution à l'étude du peuplement zooplanctonique du lac d'Ayamé (Côte d'Ivoire). Thèse de doctorat, Université Nationale de Côte d'Ivoire, $121 \mathrm{p}$.

Yté W. A., N. C. Kouassi et S. Yoro. 1996. Peuplement zooplanctonique du lac de Buyo (Côte d'lvoire) : Liste faunistique et distribution. Agro. Afr. 8 : 143 - 152. 